\title{
REZENSION
}

\section{Die Schulaufsicht als vernachlässigte Akteurin der Schulreform. Eine Sammelbesprechung}

Klein, Esther Dominique, \& Bremm, Nina (Hrsg.). (2020). Unterstützung Kooperation - Kontrolle. Zum Verhältnis von Schulaufsicht und Schulleitung in der Schulentwicklung. Wiesbaden: Springer VS, 397 S., 49,99€

Huber, Stephan Gerhard, Arnz, Siegfried, \& Klieme, Torsten (Hrsg.). (2020). Schulaufsicht im Wandel. Rollen und Aufgaben neu denken. Stuttgart: Raabe, 330 S., $43,90 €$

Die Schulaufsicht muss wieder reformiert werden. Nicht zuletzt wegen der ausbleibenden Erfolge der Reformen, die mit Nachdruck aufgrund des schlechten Abschneidens Deutschlands bei der ersten PISA-Studie eingeführt wurden. Gemeint ist vor allem die Arbeit von Schulrät*innen. Im Rahmen der Fach- und Dienstaufsicht arbeiten sie direkt mit Schulen zusammen und sollen vor Ort die bestmögliche Förderung der Schüler*innen ungeachtet ihrer Herkunft und diskriminierender Zuschreibungen gewährleisten. Erfuhr die Schulaufsicht durch die letzten Reformen einen Bedeutungsverlust, ist jetzt eine Renaissance und Neuausrichtung angeraten. So können die Beiträge in zwei kürzlich erschienenen Herausgeberbänden zur Schulaufsicht pointiert zusammengefasst werden. Da ist zum einen der von Klein und Bremm herausgegebene Band Unterstützung - Kooperation - Kontrolle. Zum Verhältnis von Schulaufsicht und
Schulleitung in der Schulentwicklung (im Folgenden mit HB1 abgekürzt). Es kommen vor allem Wissenschaftler*innen aus der Schulentwicklungs- und EducationalGovernance-Forschung zu Wort. Zum anderen vereint der von Huber, Arnz und Klieme herausgegebene Band Schulaufsicht im Wandel. Rollen und Aufgaben neu denken (im Folgenden mit HB2 abgekürzt) insbesondere Autor*innen, die direkt mit der Praxis schulaufsichtlichen Handelns befasst sind. Bemerkenswert sind die Sammelbände, da sie in seltener Weise theoretische Einordnungen, Forschungsergebnisse, Berichte aus der Praxis und Reformvorschläge zur bislang kaum erforschten Schulverwaltung bündeln.

Ich möchte die Herausgeberbände entlang von drei Punkten vorstellen. Sie bilden aus meiner Sicht die Schnittmenge der Beiträge ab. Zur Orientierung und zum Nachlesen verweise ich exemplarisch auf einzelne Beiträge. Erstens: Schulaufsicht ist nicht gleich Schulaufsicht. Diese auf den ersten Blick triviale Feststellung erweist sich dank der Beiträge von Rürup und Kuper (jeweils HB1) als zentral. Die auf einer schulrechtlichen Analyse basierenden Beiträge helfen, sich die komplexe Behördenstruktur mit ihren unterschiedlichen und bundeslandspezifischen Ebenen, Zielen und Aufgaben zu erschließen. Rürup und Kuper sensibilisieren damit noch einmal mehr dafür, dass die Mehrheit der Beiträge die Beratung zwischen Schulrät*innen und Schulleitungen und damit nur einen Teil der Aufgaben der Schulaufsicht fokussie- 
ren. Darstellungen und Analysen von weiteren Aufgaben werden nur am Rande gestreift (bspw. Auswahl von Schulleitungen, Zuweisung von Lehrkräften, Entwicklung von Lehrplänen und Stundentafeln) (kritisch dazu Berkemeyer, HB1). Mit Hilfe der Beiträge von Heinemann, Arnz und Klieme, Diedrich und Maritzen sowie Böttcher und Luig (jeweils HB2) wird diese Engführung in der Betrachtung nachvollziehbar. Demnach erodierten die Ressourcen und Aufgaben der Schulaufsicht seit den 1990er-Jahren sowohl durch die Verwaltungsmodernisierung nach Ideen des New Public Managements als auch durch die Schulautonomie-Debatte mit einer im Ergebnis ansatzweisen Aufgabenverlagerung an Schulleitungen. Die erste PISA-Studie mündete in den 2000er-Jahren zusätzlich in einen politischen Vertrauensverlust in die Arbeit der Schulaufsicht. Infolgedessen wurden neben der „klassischen“ Schulaufsicht neue Kontrollstrukturen etwa in Form von Schulinspektionen und Landesinstituten für Bildungsmonitoring eingeführt. Die zentrale Aufgabenzuschreibung durch Politik und Wissenschaft lautete für die Schulaufsicht nun, die Schulleitungen auf Basis einer breiteren Datenbasis zu beraten und $\mathrm{zu}$ unterstützen. Vor allem das Gespräch auf Augenhöhe unter Professionellen sei entscheidend, um die evidenten Probleme der Schule zu erkennen und zu lösen.

Zweitens: Die Schulaufsicht ist janusköpfig - sie soll Schulleitungen beraten und zugleich kontrollieren. Zur Annäherung an dieses Spannungsverhältnis ist die Interviewstudie von Dedering (HB1) mit Schulrät*innen hervorzuheben. Der Bei- trag zeigt, dass aus Sicht der Interviewten Beratung eine Querschnittsaufgabe in allen Aufgabenfeldern ist, wie etwa im Umgang mit erziehungsschwierigen Schüler*innen, der Schulentwicklung oder auch bei der Einstellung von Schulleitungen. Eine Weisung kommt nur zur Not zum Einsatz. Die Schulaufsicht gerät bei einer intensiven Prozessberatung zu Fragen der Schulentwicklung jedoch an ihre Kapazitätsgrenzen. Mitverantwortlich seien massive Personaleinsparungen, die die Anzahl von zu betreuenden Schulen weiter anwachsen ließen. $\mathrm{Zu}$ ähnlichen Ergebnissen aus Sicht von Schulleitungen kommt die Bremer Fragebogenstudie von Wurster, Rettinger und Feldhoff (HB1). Sie weist zudem auf die Varianz in der Einschätzung zu Beratung und Kontrolle hin. Einblicke in die konkrete Beratung bietet die Fallstudie von Herrmann (HB1). Eine Beratungssituation auf Augenhöhe im Sinne einer Reflexion zur Schärfung und eigenverantwortlichen Bearbeitung von Entwicklungsbedarfen kommt alleine durch das hierarchische Abhängigkeitsverhältnis zwischen Schulaufsicht und Schulleitung kaum zu Stande. Stattdessen geht es um eine Bestätigung von Entwicklungsprozessen, die dem Vorverständnis der Schulrät*innen von einer guten Schule entsprechen. Die Schulrät*innen nutzen dabei aber nicht wie erhofft Informationen der neu eingeführten Monitoringstrukturen und der empirischen Bildungsforschung. Vielmehr findet die Beratung auf Basis des Erfahrungswissens als ehemalige Schulleitung statt (vgl. auch Jesacher-Rößler \& Kemethofer, Rürup sowie Muslic, jeweils HB1). Die Ursachen für diese nichtintendierte Form von Beratung sehen Schmelzer und Löffler (HB2) in ei- 
ner ausbaufähigen Professionalisierung sowie in einer strukturell angelegten Rollendiffusion. Schulrät*innen wird ein schwer zu bewerkstelligender Wechsel zwischen den Kulturtechniken kontrollierender Führung und Beratung abverlangt. Je nach Rollenauslegung durch die Schulrät*innen und bundeslandspezifischen Rahmenbedingungen kann es zu qualitativen Unterschieden in der Betreuung von Schulen kommen.

Drittens: Was ist zu tun? Die Beiträge der Herausgeberbände bieten eine große Bandbreite an Reformvorschlägen. So plädiert Hochschild (HB2) für eine Übertragung der Aufgaben der Schulrät*innen auf die Ebene von Schulleitungen und Schulnetzwerken. Wenzel und Heußen (HB2) sehen die Schulaufsicht indes als wichtige Partnerin bei der Umsetzung von lokalen Bildungsnetzwerken. Hebborn (HB1) fordert die Bundesländer dazu auf, die schulaufsichtlichen Aufgaben nicht weiter zu zentralisieren, sondern vielmehr die Schulaufsicht stärker kommunal und schulformübergreifend zu organisieren (ähnlich argumentieren Rürup sowie Klein und Bremm (jeweils HB1) mit Blick auf Erfahrungen aus den USA). Röder und Manitius (HB2) treten für eine stärkere institutionelle und personelle Trennung von klassischen schulaufsichtlichen und schulentwicklungsberatenden Aufgaben ein (auch in Form einer Auslagerung an qualifizierte und zertifizierte Schulentwicklungsberater*innen). Damit verbunden weisen sie auf eine notwendige Spezifikation von Beratungsleistungen hin (bspw. dienstrechtliche Beratung von Schulleitungen in Personalentscheidungen vs. Implementation eines Teamkonzepts).
Diedrich (HB1) spricht sich mit Blick auf die wahrgenommenen funktionalen Defizite der neuen datengestützten Systemsteuerung für eine kontrollierte Grenzüberschreitung aus. Nicht Abgrenzung, sondern permanente Selbstreflexion und enge Abstimmung der Akteure ermöglichen eine nachhaltige Qualitätsentwicklung. Eine integrative Sichtweise hinsichtlich der exemplarisch vorgestellten Reformvorschläge nehmen Arnz und Klieme sowie Huber in ihren Beiträgen ein (jeweils HB2).

Insgesamt setzen die Herausgeberbände an einer wichtigen Lücke in der Beschäftigung mit einer Reform von Schule an und vertiefen die Erkenntnisse des vielbeachteten Gutachtens von Bogumil, Fahlbusch und Kuhn (2016) zur Weiterentwicklung der Schulverwaltung in NordrheinWestfalen. Sie legen den Schluss nahe, dass im Zuge der Schulreformen der 1990er- und 2000er-Jahre ein inkonsistenter Umbau der Steuerungsarchitektur der deutschen Schulsysteme stattfand, der in der Praxis zu Unsicherheiten und Spannungen, wenn nicht sogar zu einem Scheitern der Reformziele führen kann. Allerdings wird in den Bänden nur vereinzelt das grundsätzliche Reformcredo einer Verantwortungsaktivierung hinterfragt, das vom System Schule eine stärkere Übernahme von Verantwortung für Entwicklungsprozesse und deren Ergebnisse einfordert, was - wie einige Beiträge zeigen - Schulen und Schulverwaltung auch überfordern kann. Vor allem sind die beiden Bände als Kritik an der Bildungsforschung der letzten Jahre zu verstehen. Neue administrative Reformstrukturen und -prozesse, etwa im Rahmen der Einführung 
der Schulinspektion oder von Lernstandserhebungen, standen vielfach im Mittelpunkt der Forschung. Vernachlässigt wurde hingegen der Einfluss der „traditionellen" Schulverwaltung (in alter und neuer Ausrichtung) auf den Schulund Unterrichtsalltag. Neben der staatlichen ist hierzu auch die kommunale Schulverwaltung zu zählen. Die Bände verdeutlichen insgesamt den dringenden Handlungsbedarf hinsichtlich einer vielschichtigen Forschung zur Schulverwaltung. Notwendig sind dafür Studien, die über die programmatisch aufgeladene Frage hinausreichen, ob Schulrät*innen nun beraten und/oder kontrollieren. Der Fokus sollte auch stärker auf grundständige Aufgaben, wie die Lehrkräftezuweisung, sowie aktuelle Herausforderungen der Schulverwaltung gerichtet sein (bspw. Digitalisierung von Schule und Unterricht, Inklusion, Integration von Neuzugewanderten, demografischer Wandel). Das Potenzial für einen solchen Zugang zeigt der Beitrag von Hermstein, Hußmann und Vaskova (HB1) zur inklusionsorientierten Schulentwicklung durch (kommunale) Schulträger und Schulaufsicht. Erst eine breiter angelegte Schul- verwaltungsforschung ermöglicht validere Aussagen dazu, ob und wie die Schulaufsicht reformiert werden muss und welcher der in den Sammelbänden umrissenen Vorschläge dafür wichtige Ansatzpunkte bietet. Besondere Berücksichtigung sollte dabei die in den Beiträgen immer wieder aufscheinende ungleiche Unterstützung von Schulen und damit auch von Schüler*innen finden. Eine entsprechende Auseinandersetzung ist damit hoch anschlussfähig an Fragen zu schulseitigen Ursachen von Bildungsungleichheit.

\section{Literatur}

Bogumil, J., Fahlbusch, R. M., \& Kuhn, H.-J. (2016). Weiterentwicklung der Schulverwaltung des Landes NRW. Bochum: Wissenschaftliches Gutachten im Auftrag des Finanzministeriums.

Norbert Sendzik, Wissenschaftszentrum Berlin für Sozialforschung (WZB)

E-Mail: norbert.sendzik@wzb.eu

https://doi.org/10.31244/dds.2021.01.10 